\title{
Conductance of Photons and Anderson Localization of Light
}

\author{
A. A. Asatryan, ${ }^{1}$ L. C. Botten ${ }^{1}$ R. C. McPhedran ${ }^{2}$, C. M. de Sterke ${ }^{2}$, T. Langtry ${ }^{1}$ and N.A Nicorovici ${ }^{1}$ \\ ${ }^{1}$ CUDOS and Department of Mathematical Sciences, University of Technology, Sydney, Broadway, New South Weals 2007, Australia \\ ${ }^{2}$ CUDOS and School of Physics, University of Sydney, New South Weals, 2006, Australia \\ E-mail: ara.asatrian@uts.edu.au
}

\begin{abstract}
The conductance properties of photons in disordered two-dimensional photonic crystals is calculated using exact multipole expansions technique. The Landauer's twoterminal formula is used to calculate the average of the conductance, its variance and the probability density distribution. (C2003 Optical Society of America OSIC codes: 290.4210 Multiple scattering
\end{abstract}

One of the fundamental properties of wave propagation in disordered media is Anderson localization [1], the phenomenon in which the wave amplitude decreases exponentially due to strong interference between multiply scattered waves. This phenomenon was originally discovered for electrons and now is a major research area in classical wave physics. In spite of intensive research a rigorous theory of localization has not been formulated yet. Various approximate theories have been developed to describe the Anderson transition including the phenomenological scaling theory of localization [2]. This is a general theory developed to describe the localization for electrons, which was subsequently adopted to describe the Anderson transition for photons. According to this theory all waves are localized for oneand two-dimensional infinite geometries for all wavelengths even for arbitrary weak disorder, while for three-dimensional problems only a finite range of wavelengths can be localized. Localization properties can be deduced from the scaling properties of the conductance as a function of the size of the sample.

Soon after the formulation of the scaling theory, universal conductance fluctuation (UCF) phenomenon were discovered [3,4]. According to this theory, the conductance is strongly fluctuating and its variance does not depend on the degree of disorder or the size of the sample. This raises serious doubts about the applicability of the scaling theory.

Here we carry out systematic investigation of the conductance, its average and its distribution for two dimensional optical scattering systems. We consider a two dimensional photonic crystal, which we model as a set of gratings. The unit cell comprises a set of cylinders with circular cross-section, taken to be infinitely long. The conductance of photons can be calculated using Landauer's two-probe formula [5]

$$
g=\sum T_{p q} .
$$

We have carried out a comprehensive investigation of the properties of the conductance and its scaling properties for this two dimensional photonic crystal. The method based on the rigorous theory of multipole expansions [6]. We have found that while for weak disorder the variance of the conductance indeed does not depends on the size of the sample, the degree of disorder strongly influences the conductance variations, which decrease with increasing disorder. We have calculated the distribution of the conductance for the three regimes of propagation: diffusive, localized and at the transition. The distribution function of the 
conductance is Gaussian for the diffusive regime and log-normal in the Localized regime. A scaling theory for photon localization will also be presented.

\section{References}

1. P. Sheng, Introduction to Wave Scattering, Localization, and Mescscopic Penomena, (Academic, San Diego, 1995).

2. E. Abrahams, P.W.Anderson, D.C. Licciardello, and T.V. Ramakrishnan, "Scaling theory of localization: Absence of quantum diffusion in two dimensions," Phys. Rev. Lett, 42, 673-676, (1979)

3. P.A. Lee, and A.D. Stone, "Universal Conductance Fluctuations in Metals," Phys. Rev. Lett. 55, 1622-1625 (1985).

4. B.L. Altschuler, JETP Lett. 41, 648-651 (1985).

5. R. Landauer, "Electrical resistance of disordered one-dimensional lattices," Phil. Mag. 21, 863-867, (1970).

6. L.C. Botten, N.A. Nicorovici, A.A.Asatryan, R.C. McPhedran, C. Martijn de Sterke, and P.A. Robinson, "Formulation for electromagnetic scattering and propagation through grating stacks of metallic and dielectric cylinders for photonic crystal calculations.” J. Opt. Soc. Am A 17, 2165-2176 (2000). 